\title{
MÉDICOS LATINOAMERICANOS EN CATALUÑA: PROCESOS DE INTEGRACIÓN Y DESARROLLO PROFESIONAL
}

\author{
Andrés Freire* \\ Erika Masanet $^{* *}$
}

\begin{abstract}
Este artículo analiza los procesos de integración profesional de los médicos latinoamericanos en el mercado de trabajo de salud catalán, en España, así como sus posibilidades de desarrollo profesional. El artículo se basa en dos investigaciones de carácter cualitativo que han utilizado entrevistas en profundidad con médicos latinoamericanos residentes en la Comunidad Autónoma de Cataluña. Los resultados del trabajo permiten identificar diversos procesos de integración profesional de estos médicos inmigrantes, al tiempo que muestran las principales barreras que enfrentan en dichos procesos.
\end{abstract}

Palabras clave: migración internacional, médicos inmigrantes latinoamericanos, integración profesional, barreras, contexto catalán y español.

\section{Introducción}

Dentro de la creciente complejidad que presentan las migraciones internacionales contemporáneas, la movilidad de trabajadores cualificados, y específicamente la de los profesionales de la salud, ha cobrado en las últimas décadas una mayor relevancia cuantitativa y cualitativa. En el contexto internacional, España participa activamente en la movilidad de médicos, tanto como país de destino como emisor. Así, el país se ha ido configurando, principalmente desde la década de 1990, en uno de los principales destinos para médicos latinoamericanos y en menor medida de otras regiones, lo que ha supuesto una mayor diversificación de la fuerza laboral médica en

\footnotetext{
${ }^{*}$ Magister en la Universitat Autònoma de Barcelona. Barcelona/España.

** Centro de Investigação e Estudos de Sociologia, Instituto Universitário de Lisboa (CIES ISCTE-IUL). Lisboa/Portugal.
} 
cuanto a los lugares de procedencia, formación y perfil académico ${ }^{1}$. A su vez, ha mantenido un flujo emigratorio variable, principalmente dentro de la Unión Europea ${ }^{2}$, que se estaría incrementando en los últimos años de crisis socioeconómica.

Para el caso de la Comunidad Autónoma de Cataluña, ámbito geográfico en donde centramos nuestras investigaciones, los datos más recientes del año 2012 indican que en la provincia de Barcelona el $17,3 \%$ del total de médicos colegiados eran extranjeros de acuerdo al lugar de nacimiento, representando para ese año el 42,8\% de las nuevas colegiaciones. Si bien la colegiación de los médicos extranjeros en Barcelona ha seguido una tendencia creciente desde 1999, a partir de 2010 se produce un punto de inflexión en la evolución de este grupo, de tal forma que las nuevas colegiaciones de médicos extranjeros disminuyen de forma notable ${ }^{3}$.

Este crecimiento sostenido en la cantidad de profesionales que han llegado a España ha generado un creciente interés en diversos ámbitos, principalmente en torno a la gestión y planificación de los recursos humanos en salud. Sin embargo, las particularidades de sus procesos migratorios, experiencias y trayectorias profesionales, social e históricamente construidas y situadas, han sido menormente visibilizadas e investigadas en los recientes estudios migratorios desde las ciencias sociales.

En este sentido, el objetivo del presente artículo es analizar los procesos de integración profesional de los médicos latinoamericanos en el mercado de trabajo de salud catalán y sus posibilidades de desarrollo profesional, tomando como ámbito geográfico la Comunidad Autónoma de Cataluña, a partir de las percepciones de los propios médicos sobre sus trayectorias migratorias y profesionales. Para ello nos basamos en los resultados de dos investigaciones sociológicas de carácter cualitativo desarrolladas por los autores y que han tenido por objeto común de estudio la migración de médicos latinoamericanos a España. Ambas investigaciones han utilizado como técnica de recolección de datos la entrevista en profundidad, compartiendo el ámbito geográfico (Cataluña) y temporal (entre febrero y julio de 2011) del trabajo de campo.

Se realizaron un total de 24 entrevistas a 11 médicas y 13 médicos que emigraron a España a partir del año 2000 desde diversos países de

${ }^{1}$ GONZÁlEZ LÓPEZ-VALCÁRCEL, Beatriz; BARBER PÉREZ, Patricia; SUÁREZ VEGA, Rafael. Oferta y necesidad de especialistas médicos en España (2010-2025); BRUGUERA, Miquel; SOLER, Marc; CAMPS, Berenguer. Estudi demogràfic dels metges del COMB: Importància dels metges estrangers.

${ }^{2}$ GONZÁlEZ LÓPEZ-VALCÁRCEL, BARBER PÉREZ, SUÁREZ VEGA, op. cit.

${ }^{3}$ CAMPS, Berenguer. Informe sobre demografía col·legial 2012. 
Latinoamérica: Argentina, Brasil, Colombia, Cuba y Uruguay. Se trata de profesionales que residían de forma permanente en diferentes localidades de Cataluña desde hacía al menos un año al momento de la entrevista, nacidos en América Latina y formados como Licenciados en Medicina y/o como Especialistas en los países de origen, y con edades comprendidas entre los 26 y los 56 años. Las muestras incluyeron a 17 profesionales que obtuvieron su especialidad en el extranjero en distintas áreas ${ }^{4}, 6$ médicos que se estaban formando como especialistas en España a través del sistema de formación "MIR" ${ }^{5}$ y un médico generalista. En el presente artículo se exponen los resultados del análisis temático de las entrevistas en base a las siguientes categorías de análisis: inserción profesional en el mercado de trabajo y el sistema sanitario español, proceso de homologación de la titulación en Medicina y de la especialidad, y percepciones de prejuicios y discriminación.

\section{Contexto general de la migración de médicos de América Latina a \\ España}

En las últimas décadas se asiste a un aumento significativo de la migración internacional de los profesionales de la salud en la mayoría de los países de la OCDE, algunos de los cuales se consolidan como destinos tradicionales, mientras que otros se configuran como países emisores a la vez que receptores ${ }^{6}$.

Dentro de un contexto mundial de escasez y distribución geográfica desigual de trabajadores de este sector ${ }^{7}$ y un mercado laboral sanitario cada vez más internacionalizado y liberalizado, la movilidad internacional de médicos se ha planteado como solución a corto plazo para los Estados y sistemas de salud de los países centrales y semi-periféricos, junto a otras medidas implementadas para regular su cantidad (retraso jubilatorio, aumento del numerus clausus, mejora de las condiciones laborales) ${ }^{8}$. Así, las necesidades de estos países con una mayor capacidad para atraer y reclutar a los migrantes más cualificados, en el marco de políticas migratorias selectivas y restrictivas

\footnotetext{
${ }^{4}$ Las especialidades de los entrevistados correspondieron a: Anestesiología, Cirugía, Geriatría, Medicina Familiar, Medicina Interna, Nefrología, Oftalmología, Oncología, Pediatría, Psiquiatría, Radiodiagnóstico y Traumatología.

${ }^{5}$ MIR, Médico Interno Residente, se refiere al sistema español de formación de médicos especialistas.

${ }^{6}$ DUMONT, Jean-Christopher; ZURN, Pascal. Immigrant health workers in OECD countries in the broader context of highly skilled migration.

7 TAYLOR, Allyn L.; DHILLON, Ibadat S. The WHO Global Code of Practice on the International Recruitment of health personnel: The evolution of Global Health Diplomacy.

${ }^{8}$ DUMONT, ZURN, op. cit.
} 
a la movilidad de personas ${ }^{9}$, han generado una demanda determinante en la conformación, direccionalidad y desarrollo de estos flujos migratorios.

En el caso de los médicos que han emigrado desde América Latina a España en los últimos años, esta movilidad se ha visto facilitada por factores de distinto orden (social, cultural, histórico, económico y político), con una actuación importante de diversos actores públicos (principalmente el Estado) y privados en la determinación de estos desplazamientos. En tal sentido, pueden señalarse las limitaciones de los mercados de trabajo, condiciones laborales y oferta formativa local en origen; idioma común y proximidad cultural (a partir de vínculos históricos-estructurales poscoloniales entre regiones); la actuación de agencias y el reclutamiento institucional; las políticas y acuerdos estatales de otorgamiento de visas y el reconocimiento de títulos académicos y acuerdos entre instituciones educativas, en el marco de múltiples convenios y tratados económicos y culturales de cooperación ${ }^{10}$.

Asimismo, una movilidad de larga data intensificada en los últimos años, corresponde a la protagonizada por médicos que se trasladan desde Latinoamérica a España para realizar la formación especializada y otras formaciones de posgrado o prácticas académico-profesionales, consolidándose como una de las vías más importantes para la llegada de este colectivo ${ }^{11}$. De esta manera, estas prácticas transnacionales institucionalizadas, en principio correspondientes a movilidades temporales, se han convertido en otro de los principales factores para el desarrollo de la migración cualificada en el contexto de una mayor internacionalización de los sistemas de educación superior ${ }^{12}$.

Algunas investigaciones han evidenciado una serie de motivaciones para la emigración del personal de salud desde diversas regiones latinoamericanas ${ }^{13}$. En tal sentido se destacan la insatisfacción con las condiciones laborales (bajos salarios y pocas posibilidades de desarrollo profesional, situaciones de pluriempleo, jornadas extensas, inseguridad

\footnotetext{
${ }^{9}$ CASTLES Stephen. The forces driving global migration.

10 ORGANIZACION PANAMERICANA DE LA SALUD. Migración de recursos humanos en salud. Estudio Subregión Andina.

${ }^{11}$ GONZÁLEZ LÓPEZ-VALCÁRCEL, BARBER PÉREZ, SUÁREZ VEGA, op. cit.

${ }^{12}$ PELLEGRINO, Adela. ¿Drenaje o éxodo? Reflexión sobre la migración cualificada.

${ }^{13}$ ORGANIZACION PANAMERICANA DE LA SALUD, op. cit.; PELLEGRINO, Adela; BENGOCHEA, Julieta. Informe descriptivo y analítico sobre las características sociales, demográficas y motivacionales de los egresados de Medicina y Enfermería migrantes y no migrantes; FREIRE, Andrés. Migración cualificada: trayectorias socioprofesionales de los médicos latinoamericanos en Cataluña; MASANET, Erika. A imigração qualificada dos(as) médicos(as) brasileiros(as) na Espanha e em Portugal: quais as diferenças e as semelhanças? Un conjunto de informes a nivel local que forman parte del proyecto de investigación "Migraciones para el desarrollo compartido" (OMSOPS-EASP-MSP) pueden consultarse en: <http://www.mpdc.es/>.
} 
laboral y carencias en la infraestructura), la baja calidad de vida en contextos regionales de crisis económicas y diversos grados de conflictividad social y política; las escasas oportunidades formativas, académicas y laborales y la falta de reconocimiento social de la actividad profesional. Se señala además la búsqueda de oportunidades para acceder a una mejor remuneración y formación, de trabajar en sistemas de salud de mayor calidad y recursos para el desarrollo de la práctica profesional, así como de mejorar la conciliación entre vida familiar y laboral.

Además de la combinación de los referidos motivos y factores y del papel de las redes sociales transnacionales, formales e informales, deben tenerse en cuenta los factores contextuales de destino para un mejor análisis de los determinantes de la migración. En tal sentido, puede señalarse la generación de una demanda del mercado de trabajo médico español y de los sistemas de salud autonómicos de incorporar a médicos inmigrantes, tanto desde el sector público como el privado, y principalmente en algunos lugares de trabajo y especialidades concretas. Así es que se ha apuntado un "déficit selectivo" de médicos para el conjunto de España ${ }^{14}$ en un mercado laboral sanitario segmentado geográficamente y por especialidades y un sistema nacional de salud fuertemente descentralizado ${ }^{15}$.

Ciertos autores ${ }^{16}$ han señalado además, que algunas de estas plazas que no se llegan a ocupar con médicos autóctonos de acuerdo a ciertos "niveles de aceptabilidad"- en cierta medida por los altos niveles de precariedad, las menores posibilidades de desarrollo profesional o el poco prestigio profesional que suponen - han sido cubiertas generalmente con médicos extranjeros, en su mayoría extracomunitarios.

Las necesidades de médicos han respondido tanto a la falta de planificación e ineficiente gestión de los recursos humanos disponibles ${ }^{17}$, como a una mayor inversión pública y demanda privada en servicios de salud, escenario que está cambiando a partir de la reducción del gasto público sanitario en los últimos años en el contexto de la agudización de la crisis económica ${ }^{18}$.

\footnotetext{
${ }^{14}$ ORGANIZACIÓN MEDICA COLEGIAL. Demografía Médica. Apuntes para la eficiencia del Sistema Sanitario.

15 GONZÁLEZ LÓPEZ-VALCÁRCEL, Beatriz; BARBER PÉREZ, Patricia. Los recursos humanos y sus desequilibrios mitigables.

${ }^{16}$ Ibidem; BRUGUERA, SOLER, CAMPS, op. cit.

${ }^{17}$ BRUGUERA, SOLER, CAMPS, op. cit.; GONZÁLEZ LÓPEZ-VALCÁRCEL, Beatriz; BARBER PÉREZ, Patricia; DÁVILA QUINTANA, Carmen D. Opportunities in an expanding health service: Spain between Latin American and Europe.

${ }^{18}$ GONZÁlEZ LÓPEZ-VALCÁRCEL, BARBER PÉREZ, DÁVILA QUINTANA, op. cit.
} 
En otro orden, los médicos inmigrantes conforman, como migrantes cualificados, un grupo con ciertas ventajas en comparación con otros grupos de migrantes, debido a la existencia de ciertas facilidades (administrativas, legales, institucionales) durante su movilidad y proceso migratorio ${ }^{19}$. Sin embargo, la pertenencia a este grupo no les exime de enfrentar en sus trayectorias, una serie de obstáculos y limitaciones durante su proceso de inserción laboral e integración profesional. En este sentido, algunos autores ${ }^{20}$ ya advertían de que si bien el migrante con altos niveles de formación y experiencia acumulada posee algunas ventajas, como mayores facilidades de adaptación e integración al nuevo entorno y una más rápida movilidad social y espacial, éstas no aseguran una inserción laboral exitosa o una integración completa en las sociedades de destino, ni evitan algunos de los desafíos que supone la incorporación a la que se enfrentan el conjunto de inmigrados.

\section{Procesos de inserción e integración profesional}

El análisis cualitativo permite identificar, en la sociedad de instalación, la existencia de diversas condiciones de inserción e integración y posibilidades de desarrollo profesional en las trayectorias de los médicos latinoamericanos entrevistados. Dentro de esta diversidad de experiencias y trayectorias profesionales, no obstante, podemos identificar a grandes rasgos dos grupos o perfiles principales de médicos a partir del tipo de inserción o incorporación profesional que han tenido, como "punto de partida" en la sociedad de destino: por un lado, aquellos que llegaron para formarse como especialistas a través del sistema de formación "MIR", y por otro, los que se incorporaron directamente al mercado laboral médico.

En el primer grupo, la migración se vinculó en principio a proyectos migratorios temporales en España y Cataluña, determinados inicialmente por la duración de la formación. Para aquellos médicos sin título de especialista, la decisión de formarse en España a través del sistema MIR se debió principalmente a las ventajas de las condiciones de esta formación especializada frente a la que podían acceder en gran parte de sus países de origen (u otros destinos tradicionales para los profesionales latinoamericanos como Estados Unidos), tales como las mayores facilidades en el acceso a esta formación, la calidad de sus programas y centros de investigación y, muy especialmente, la satisfactoria remuneración salarial: "Y tú cuando te

\footnotetext{
${ }^{19}$ PELLEGRINO, Adela; VIGORITO, Andrea. La emigración calificada desde América Latina y las iniciativas nacionales de vinculación.

${ }^{20}$ FAVELL, Adrian; FELDBLUM, Miriam; PETER SMITH, Michael. The Human Face of Global Mobility: A Research Agenda.
} 
vas a formar [como especialista] no tienes que pagar como allá sino que te pagan y lo único que tienes que hacer es pasar un examen de acceso" (MIR, Colombia). Otro entrevistado manifestaba: "En Uruguay te pagan pero una miseria, un tercio de lo de acá. En Chile que es un destino interesante por ejemplo tienes que pagar para hacer la especialidad" (MIR, Uruguay).

En la mayoría de los casos del grupo de médicos que llegó para especializarse, dicha formación actuó como una primera inserción en el mercado de trabajo sanitario español que les facilitó una adaptación gradual y una socialización profesional y familiarización con el nuevo entorno, al tiempo que les permitió generar una serie de redes y contactos profesionales con colegas e instituciones sanitarias en el nuevo contexto profesional y social. Todo ello facilitó, posteriormente, el acceso a contratos de trabajos muchas veces en las mismas instituciones donde realizaron la residencia médica o bien en otros centros donde habían establecido contactos previos.

La realización de la formación especializada en España supondría, además, un modo de incorporación diferencial en el mercado de trabajo, con ciertas ventajas, ya que implica que esa formación especializada (título de especialista) y la experiencia laboral, como parte de su capital cultural, no necesitan ser reproducidas o convalidadas en la sociedad de destino. Este conjunto de factores contribuiría a una mayor igualdad con los médicos autóctonos en las oportunidades laborales y profesionales y, por ende, incrementaría las posibilidades de una mejor carrera e integración profesional.

El segundo grupo de médicos está formado por aquellos que emigraron desde América Latina a España y que se incorporaron directamente al mercado de trabajo médico (en su gran mayoría a partir de ofertas concretas de trabajo) y que, a diferencia de aquellos que llegaron inicialmente a formarse, se asocian mayoritariamente a proyectos de permanencia en la sociedad de instalación, en varios casos correspondientes a proyectos migratorios familiares. Son, según nuestro análisis, los que mayores dificultades o barreras han encontrado en su proceso de integración profesional.

Este grupo de entrevistados incluye tanto a médicos especialistas formados como tales en el extranjero como a médicos generalistas. Para estos médicos, en algunos casos reclutados en origen, un factor determinante para haber emigrado ha sido una oferta que implicaba mejorar las condiciones de trabajo y, en menor medida, una mejora económica que generaba a la vez expectativas de obtener una mejor calidad de vida para sí y sus familias.

De acuerdo a las entrevistas, en general, la incorporación inicial en el mercado de trabajo de este grupo se ha producido, por necesidad o estrategia 
(e independientemente si lograron o no una temprana movilidad laboral) en determinados espacios laborales del sector salud, a saber: los servicios de urgencias de los hospitales (tanto del sistema público, privado o concertado), en empresas privadas subcontratadas que prestan servicios para el sistema de emergencias de Cataluña, en el área de Medicina del Trabajo (Mutuas) y en hospitales o centros de atención más alejados de las capitales provinciales.

En referencia a estas plazas, los médicos entrevistados coinciden en señalar que se trata de puestos de trabajo en general "poco atractivos" por el mayor ritmo de trabajo y el "desgaste" que suponen, las escasas posibilidades de desarrollo profesional que ofrecen y el bajo estatus y prestigio que otorgan, así como por las condiciones de mayor precariedad laboral. Estas características explicarían el mayor desinterés de los médicos autóctonos a ocupar dichas plazas y la elevada presencia de médicos extranjeros.

Tú vas a los servicios de urgencias acá y la mayoría son extranjeros, la mayoría de los que trabajan son latinos. Nadie las quiere escoger, son las últimas que la gente quiere escoger. Todo el mundo quiere como algo más relajado, más tranquilo, en vez de estar corriendo... Pero muchos médicos latinos encuentran allí la posibilidad de trabajar (Especialista).

Dichos espacios laborales son considerados por la mayoría de los médicos como una "puerta de entrada" al mercado laboral español y, en general, son visualizados como una etapa transitoria en las trayectorias laborales, ya que brindan oportunidades de rápida inserción laboral posibilitando, a su vez, una socialización y experiencia inicial y la generación de contactos profesionales.

En tal sentido, es importante aclarar que aunque la gran mayoría de este grupo de médicos se incorporó inicialmente a estos espacios, varios de ellos lograron una movilidad laboral que les permitió acceder a mejores puestos de trabajo en cuanto a las condiciones salariales, los horarios, la estabilidad laboral, la ubicación geográfica de los centros y, en menor medida, a posibilidades de desarrollo profesional.

\section{Barrera institucional: la homologación de la especialidad médica}

Una de las principales barreras a la que se enfrentan los médicos que se han formado como especialistas en el extranjero y que llegan a trabajar a España en el contexto espacial y temporal analizado, son las grandes dificultades en torno al reconocimiento administrativo del título de especialista, gestión actualmente dependiente del Ministerio de Sanidad, Política Social e Igualdad español.

En comparación con el proceso de homologación del título de Licenciado en Medicina que suele tratarse de un procedimiento rápido y 
sin mayores dificultades para la mayoría de médicos latinoamericanos, de acuerdo a los entrevistados el reconocimiento de la especialidad médica en España es un proceso poco transparente y lento y con niveles de exigencia muy altos de los comités de evaluación de los organismos competentes, que ha llevado además a un elevado número de resoluciones negativas, tanto bajo la anterior normativa (Orden de 14 de octubre de 1991) como con la actual (Real Decreto 459/2010, de 16 de abril). Así, casi la totalidad de los médicos especialistas manifestó al momento de la entrevista no haber conseguido aún la homologación de su especialidad o haber tenido grandes dificultades para hacerlo desde que inició el proceso.

La nueva normativa de reconocimiento de títulos de especialistas aprobada en $2010^{21}$ introdujo la novedad de proporcionar, a través de un acceso especial, una vía de solución a los médicos que ya venían ejerciendo como especialistas dentro del sistema sanitario sin tener el título homologado, además de establecer un sistema de reconocimiento más dinámico, ágil y estructurado. Sin embargo, a pesar de estas supuestas mayores facilidades para el reconocimiento de la especialidad, existe un alto número de resoluciones negativas de acuerdo a lo manifestado recientemente por algunas asociaciones de médicos extranjeros ${ }^{22}$.

Según se desprende del análisis de las entrevistas, este largo proceso de reconocimiento de la especialidad ha incidido, en general, de manera negativa en las trayectorias profesionales y las posibilidades de movilidad y desarrollo profesional de los médicos, en algún caso dificultando la incorporación al mercado de trabajo médico: "Mi principal limitante en un principio fue la homologación. Me consumió tiempo y me significó 4 o 5 años de inactividad" (Especialista).

Debido a esta situación, los médicos a los que aún no se les ha homologado el título consideran encontrarse en una situación de desventaja y desigualdad a la hora de acceder a determinadas plazas, a la vez que les coloca en una situación de mayor vulnerabilidad e inseguridad laboral en sus actuales puestos de trabajo en el nuevo contexto de crisis.

Por ello, la obtención de la homologación es considerada como un factor imprescindible para la igualdad de oportunidades y la movilidad profesional. En base a esta barrera, la gran mayoría de los médicos especialistas ha experimentado un proceso de descalificación profesional (deskilling) o una movilidad profesional descendente que ha implicado un retroceso en

\footnotetext{
${ }^{21}$ Puede consultarse en: < http://www.boe.es/boe/dias/2010/05/03/pdfs/BOE-A-2010-6960.pdf>.

${ }^{22}$ Cf. Nota de prensa: <http://www.gacetamedica.com/gaceta/articulo.aspx?idart=752604\&idcat= $797 \&$ tipo $=2>$.
} 
la posiciones ocupadas y la pérdida de su estatus profesional en relación al que tenían en sus países de origen, realizando en algunos casos tareas o actividades por debajo de su cualificación o de menor complejidad dentro de su especialidad: "Yo trabajo sin el título homologado, pero eso no es legal [....]. Entonces, a nosotros nos ponen a hacer actividades que perfectamente podría hacer un médico general, tareas sin complejidad mayor" (Especialista no homologado).

Una parte de estos médicos se encuentra en una situación por lo menos paradójica ya que han sido contratados para trabajar como especialistas en el sector público y privado y ejercen actividades propias de su especialidad, a la vez que no se les ha homologado su título. Los obstáculos legales para reconocer su titulación de especialista y las necesidades de personal en determinadas especialidades, les ha llevado a ser contratados bajo esas circunstancias, en algunos casos con una menor retribución económica.

Aquí no tengo homologada mi especialidad y trabajo como especialista. Esto suele pasar. [...] Yo cobro como médica general porque no tengo la especialidad homologada aquí aún. Yo cobro menos que una especialista con título, aunque ejerzo como especialista (Especialista no homologado).

Es menester señalar que, ante las dificultades descritas por los médicos inmigrados para homologar su especialidad y, por tanto, de reproducir su capital educativo en destino, algunos han optado por realizar una segunda especialidad en España, como estrategia de recualificación, a fin de lograr un mejor posicionamiento en sus carreras profesionales.

\section{Prejuicios y discriminación}

Otro de los obstáculos a los que varios médicos entrevistados se han enfrentado en destino y que ha incidido negativamente en sus procesos de integración y desarrollo profesional, son los prejuicios y la discriminación indirecta, y en menor medida directa, en el ámbito profesional y práctica laboral diaria.

Según los entrevistados, existe un prejuicio negativo sobre la formación recibida en los países de origen, identificadas erróneamente como de "baja calidad" (aún en los casos en donde poseen el título homologado) dado su lugar de procedencia y su condición de "latinoamericanos" o "sudamericanos". En tal sentido, algunos médicos entrevistados, especialmente el grupo de especialistas formados en origen, han percibido cierta desconfianza profesional e infravaloración de sus competencias formativas y técnicas por 
parte de sus jefes, supervisores, colegas autóctonos, y en menor medida del personal no médico y pacientes, sobre todo al inicio de su actividad laboral.

Algunos colegas y pacientes creen que si un médico es latinoamericano no está bien preparado y eso no es así. Lo que pasa que algunos tienen una formación más práctica y menos teórica que muchos médicos de aquí, aunque en algunas especialidades eso también lo valoran. (Especialista).

Porque eres extranjero y esto ya da desconfianza. Pero cuando se comparan los dos curriculum te escogen a ti porque eres mejor, pero si fuesen igual(es) escogerían al otro porque el otro tiene un plus por ser de aquí. Tú tienes una desventaja: eres un extranjero. Comparativamente tienes que demostrar una valía diferente. Eso segurísimo. (Especialista).

Asimismo, algunos médicos identifican que este prejuicio sobre su formación o experiencia y el trato desfavorable o discriminatorio que se deriva, es en cierta medida independientemente de dónde se han formado o trabajado, ya que creen que se explica por su origen foráneo ("venir de fuera", "ser extranjero") y por su condición "visible" de "extranjeros", en donde se construyen diferencias a partir de los rasgos físicos y acentos. Esta situación los ubicaría en una posición de desventaja en el mercado de trabajo, limitando las oportunidades y posibilidades de desarrollo profesional:

Seguramente a ti te juzgan más duro que a todos los demás y seguramente yo me equivoco con algo y ya van a decir que es por negro. (...) Cuando tú eres diferente desconfían de ti, si tú eres igual a todo el mundo serás peor, tú tienes que ser mejor que las personas que tienes al lado para que te traten igual y eso es lo que yo he conseguido. (Especialista).

Entre extranjeros buscarán al que sea más idóneo para el puesto. Pero si es un médico extranjero que compite con uno de acá elegirán al de acá. [...] Eso porque somos latinoamericanos, aunque no lo digan de frente te dirán bien, está homologado y habla catalán, pero te dirán si eres de aquí o estás nacionalizado. La última excusa es que "tú no eres de aquí". (Especialista).

Cabe destacar dos casos de profesionales de larga experiencia y trayectoria, ambos con títulos de especialista homologados, que refieren en sus relatos a situaciones de xenofobia en los lugares de trabajo. Mientras que en un caso ha significado quedar excluido de realizar determinadas tareas en su especialidad, sufriendo un proceso de descualificación que ha condicionado sus posibilidades de desarrollo, en el otro ha supuesto ser sustituido en el cargo porque, según le manifestaron sus superiores y en 
palabras textuales, esa plaza debía ser ejercida por "una persona de aquí" en relación a "un español". En ambos casos, los entrevistados no identificaron ninguna razón objetiva que pudiera justificar tales decisiones.

\section{Conclusiones}

Los resultados presentados han ofrecido una aproximación a las experiencias profesionales de los médicos latinoamericanos en la sociedad de destino, contribuyendo al conocimiento sobre la inmigración reciente del personal médico en España, tema escasamente abordado en el contexto español.

A partir del análisis podemos constatar la existencia de procesos y modos diferenciados de integración profesional a partir de las condiciones iniciales de incorporación al mercado de trabajo y sistema sanitario que determinan diferentes oportunidades profesionales para los médicos inmigrados, entre los cuales el grupo de los formados en España (MIR) presenta unas condiciones más favorables para su posicionamiento en el mercado de trabajo e integración profesional en la sociedad de destino.

En la literatura reciente sobre el tema es común la referencia a las diversas barreras u obstáculos, formales e informales, de carácter material y simbólico, a las que se enfrentan los médicos inmigrados en sus procesos de integración profesional y durante sus trayectorias en los países de destino ${ }^{23}$. En esta línea, los resultados obtenidos permiten identificar la existencia de algunas barreras específicas para el caso del colectivo de médicos latinoamericanos en el contexto catalán y español.

En primer lugar, las dificultades en torno al reconocimiento administrativo del título de especialista en España constituyen uno de los desafíos más importantes a los que se enfrentan los médicos formados como especialistas en Latinoamérica en el contexto analizado. Como consecuencia de esta barrera institucional que impide la reproducción de parte de su capital cultural en destino, se produce un proceso de descualificación profesional o deskilling, significando el proceso migratorio una ruptura en la continuidad de sus carreras profesionales.

En segundo lugar, los entrevistados perciben que existe una barrera formada por los prejuicios y discriminación en el ámbito profesional a

\footnotetext{
${ }^{23}$ BOURGEAULT, Ivy L. et alii. Brain gain, drain \& waste: the experiencies of internationally Educated Health Professionals in Canadá; CHEN, Peggy G. et alii. Professional Experiences of International Medical Graduates Practicing Primary Care in the United States; WOLANIK, Katarzyna; ÖHLANDER, Magnus. A troubled elite? Stories about migration and establishing professional-ism as a Polish doctor in Sweden; RIBEIRO, Joana Sousa. Migration and occupational integration: foreign health professionals in Portugal.
} 
partir de la desconfianza e infravaloración en los lugares de trabajo de las competencias adquiridas en los países de origen, barrera en parte superable en el tiempo. Pero además, algunos médicos han relatado haber sido objeto de discriminación por su condición de "extranjeros" e inmigrados, su fenotipo y acento. La discriminación directa e indirecta que enfrentan los médicos inmigrantes en el lugar de trabajo también ha sido documentada en otras investigaciones realizadas en el contexto español ${ }^{24}$, norteamericano ${ }^{25}$ y europeo ${ }^{26}$. Es menester aclarar que estas formas de discriminación social interactúan con otras formas de discriminación (en función del género, la edad, etc.), algunas de las cuales pueden compartir con los profesionales autóctonos o con otros colectivos de inmigrados.

La evidencia empírica sugiere además que las trayectorias profesionales de los médicos latinoamericanos inmigrados pueden seguir un curso favorable en la sociedad de destino a partir de la superación de barreras administrativas e institucionales, la generación de mayor capital social, y ciertas estrategias de recualificación (reskilling) orientadas a la movilidad profesional. Así, una perspectiva longitudinal permitiría obtener un panorama más completo del proceso de integración profesional y social y de la evolución que han seguido estas trayectorias en contextos cambiantes y dinámicos.

Por último, cabe indicar que el actual panorama de crisis socioeconómica en España podría estar planteando un nuevo escenario en cuanto a la movilidad de profesionales de la salud entre las regiones de estudio, emergiendo así dos fenómenos simultáneos. En primer lugar, un aumento en los últimos años de la cantidad de médicos formados en España que emigran al exterior en busca de mejores condiciones laborales y oportunidades profesionales. Paralelamente, la disminución de los flujos de entrada de médicos que Ilegan a trabajar, principalmente provenientes de América Latina, debido a la mejora de las condiciones laborales en los países de origen, la reducción de la demanda de profesionales en el mercado laboral español y la significativa disminución del número de plazas disponibles para extracomunitarios en el programa de formación especializada "MIR"27. En

${ }^{24}$ CASELLAS LÓPEZ, Lorenzo; GONZÁLEZ ROJAS, Andrea; CANTOS VICENT, Raquel. MPDC. El caso de España. Producto 1. Informe sobre factores motivacionales.

${ }^{25}$ CHEN et alii, op. cit.; TOLBERT COOMBS, Alice A; KING, Roderick K. Workplace discrimination: experiences of practicing physicians.

26 RIBEIRO, op. cit.; WOLANIK, ÖHLANDER, op. cit.; COOKE, Lorelei; HALFORD, Susan; LEONARD, Pauline. Racism in the medical profession: the experience of UK Graduates.

${ }^{27}$ Para los cambios en la normativa de la Formación Especializada véase "Novedades de las órdenes de convocatoria de pruebas selectivas para el acceso a la FSE 2013/2014" del Ministerio de Sanidad, Servicios Sociales e Igualdad (<http://sis.msssi.es/fse/FicherosMirweb/QE-00/QE-02/0/ NOVEDADESCONVOCATORIAS201366.pdf $>$ ). 
la determinación de estas movilidades cabe destacar, además, la reciente implementación de programas o planes de captación de profesionales de la salud, nacionales o extranjeros, en algunos países latinoamericanos ${ }^{28}$.

\section{Bibliografía}

BOURGEAULT, Ivy Lynn; NEITERMAN, Elena; LEBRUN, Jane; VIERS, Ken; WINKUP, Judi. Brain gain, drain \& waste: the experiences of internationally Educated Health Professionals in Canada. Ottawa: University of Ottawa, 2010. Disponible en: <http://www.threesource.ca/documents/February2011/brain_drain.pdf>. Acceso en: 18.06.2013.

BRUGUERA, Miquel; SOLER, Marc; CAMPS, Berenguer. Estudi demogràfic del COMB. Importància dels metges estrangers. Barcelona: Gabinet d'Estudis Col·legials, Collegi Oficial de Metges de Barcelona, 2007.

CAMPS, Berenguer. Informe sobre demografia col/legial 2012. Barcelona: Gabinet d'Estudis Collegials, Collegi Oficial de Metges de Barcelona, 2013.

CASELLAS LÓPEZ, Lorenzo; GONZÁLEZ ROJAS, Andrea; CANTOS VICENT, Raquel. Migración de Profesionales de la Salud entre América Latina y Europa. Creación de Oportunidades para el Desarrollo Compartido (MPDC). El caso de España. Producto 1. Informe sobre factores motivacionales. Madrid: CSE-EASP, 2011.

CASTLES, Stephen. The forces driving global migration. Journal of Intercultural Studies, v. 34, n. 2, 2013, p. 122-140.

CHEN, Peggy Guey-Chi; NUNEZ-SMITH, Marcella; MAY BERNHEIM, Susannah; BERG, David; GOZU, Aysegul; CURRY, Leslie Ann. Professional Experiences of International Medical Graduates Practicing Primary Care in the United States. Journal of General Internal Medicine, v. 25, n. 9, 2010, p. 947-53.

COOKE, Lorelei; HALFORD, Susan; LEONARD, Pauline. Racism in the medical profession: the experience of UK Graduates. London: British Medical Association, Health Policy and Economic Research Unit, 2003.

DUMONT, Jean Christophe; ZURN, Pascal. Immigrant health workers in OECD countries in the broader context of highly skilled migration. In OCDE. International Migration Outlook. Paris: SOPEMI, 2007, p. 161-200. Disponible en: < http:// www.oecd.org/dataoecd/22/32/41515701.pdf>. Acceso en: 23.07.2013.

FAVELL, Adrian; FELDBLUM, Miriam; PETER SMITH, Michael. The Human Face of Global Mobility: A Research Agenda. In PETER SMITH, Michael, FAVELL, Adrian (eds.). The Human Face of Global Mobility. International Highly Skilled Migration in Europe, North America and the Asia-Pacific. New Brunswick, NJ: Transaction Press, 2006, v. 8, p. 1-25.

\footnotetext{
${ }^{28}$ Por ejemplo, el caso de Brasil el Programa "Mais Médicos para o Brasil": <http://portalsaude. saude.gov.br/portalsaude/index.cfm?portal $=$ pagina.visualizarTexto\&codConteudo $=11675 \&$ co dModuloArea $=1055 \&$ chamada=sobre-o-mais-medicos $>$; y el de Ecuador llamado "Ecuador Saludable Vuelvo Por Ti": <http://www.salud.gob.ec/ecuador-saludable-vuelvo-por-ti-2/> .
} 
FREIRE, Andrés. Migración cualificada: trayectorias socioprofesionales de los médicos latinoamericanos en Cataluña. In Actas del VII edición del Congreso sobre las Migraciones Internacionales en España. Bilbao: Universidad del País Vasco- Euskal Herriko Unibertsitatea, 2012. Disponible en: < https://dl.dropboxusercontent. com/u/1319656/Congreso_Migraciones2012/Area_03/03_2_5.pdf >. Acceso en: 30.07.2013.

GONZÁLEZ LÓPEZ-VALCÁRCEL, Beatriz; BARBER PÉREZ, Patricia. Los recursos humanos y sus desequilibrios mitigables. Gaceta Sanitaria, v. 20 (Supl 1), 2006, p.103-109.

GONZÁLEZ LÓPEZ-VALCÁRCEL, Beatriz; BARBER PÉREZ, Patricia; SUAREZ VEGA, Rafael. Oferta y necesidad de especialistas médicos en España (2010-2025). Las Palmas de Gran Canaria: Universidad de Las Palmas de Gran Canaria, 2011.

GONZÁlEZ LÓPEZ-VALCÁRCEL, Beatriz; BARBER PÉREZ, Patricia; DÁVILA QUINTANA, Carmen Delia. Opportunities in an expanding health service: Spain between Latin American and Europe. In WISMAR, Matthias, MAIER, Claudia B., GLINOS, Irene A., DUSSUALT, Gilles, FIGUERAS, Josep (eds.). Health Professional Mobility and Health Systems Evidence from 17 European countries. Brussels: European Observatory of Health Systems, 2011, p. 263-294.

MASANET, Erika. A imigração qualificada dos(as) médicos(as) brasileiros(as) na Espanha e em Portugal: quais as diferenças e as semelhanças?. In PADILLA, Beatriz; RODRIGUES, Elsa; MASANET, Erika; FERNANDES, Gleiciani; GOMES, Mariana; FRANÇA, Thais (orgs.). Novas e velhas configurações da imigração brasileira na Europa. Atas do Segundo seminário sobre imigração brasileira na Europa. Lisboa: ISCTE, 2012, p. 159-174.

OECD. International Migration of Health Workers. Improving international Cooperation to address the global health workforce crisis. París: OECD Policy Brief, 2010. Disponible en: <http://www.oecd.org/dataoecd/8/1/44783473.pdf>. Acceso: 1.05. 2013.

ORGANIZACIÓN MÉdICA COLEGIAL DE ESPAÑA. Demografía Médica. Apuntes para la eficiencia del Sistema Sanitario. 2009. Disponible en: <https://www. cgcom.es/sites/default/files/Libro\%20demografia\%20copia_0.pdf>. Acceso en: 08.06.2013.

ORGANIZACIÓN PANAMERICANA DE LA SALUD. Migración de recursos humanos en salud. Estudio Subregión Andina. Lima: OPS, 2006. Disponible en: <http:// new.paho.org/hq/dmdocuments/2010/RH_Migracion_RHUS-Subregion_Andina. pdf>. Acceso en: 26.07.2013.

ORGANIZACIÓN PANAMERICANA DE LA SALUD. Migración calificada en salud, impacto financiero, reconocimiento de títulos. Retos y perspectivas en los países de la Región Andina. Lima: OPS, 2013.

PELLEGRINO, Adela. ¿Drenaje o éxodo? Reflexión sobre la migración cualificada. Montevideo: Universidad de la República - Facultad de Ciencias Sociales Programa Población, 2001. 
PELLEGRINO, Adela; BENGOCHEA, Julieta. Informe descriptivo y analítico sobre las características sociales, demográficas y motivacionales de los egresados de Medicina y Enfermería migrantes y no migrantes. Montevideo: Unidad Multidisciplinaria, FCS, UDELAR, 2010.

PELLEGRINO, Adela, VIGORITO, Andrea. La emigración calificada desde América Latina y las iniciativas nacionales de vinculación. Un análisis del caso uruguayo. Revista Pensamiento Iberoamericano, v. 4, n. 1, 2009, p.189-215.

RIBEIRO, Joana Sousa. Migration and occupational integration: foreign health professionals in Portugal. In KUHLMANN, Ellen; SAKS, Mike (eds.). Rethinking professional governance: International directions in health care. Bristol: The Policy Press, 2008, p. 201-215.

TAYLOR, Allyn L.; DHILLON, Ibadat S. The WHO Global Code of Practice on the International Recruitment of health personnel: The evolution of Global Health Diplomacy. Global Health Governance, v. V, n. 1, 2011, p 1-24.

TOLBERT COOMBS, Alice A; KING, Roderick K. Workplace discrimination: experiences of practicing physicians. Journal of the National Medical Association, v. 97, n. 4, 2005, p. 467-477.

WOLANIK BOSTRÖM, Katarzyna; ÖHLANDER, Magnus. A troubled elite? Stories about migration and establishing professionalism as a Polish doctor in Sweden. In FAIST, Thomas (ed). Working Papers - Center on Migration, Citizenship and Development (COMCAD), n. 110. Bielefeld: COMCAD, 2012, p. 1-16.

\section{Abstract}

\section{Latin American doctors in Catalonia: integration process and professional development}

This article analyzes the professional integration of Latin American doctors working in the Catalan health labor market, in Spain, as well as their professional development opportunities. The article is based on two qualitative researches that have used in depth interviews with Latin American doctors who live in the Autonomous Community of Catalonia. The research results allow identifying diverse professional integration processes of these immigrant doctors, while showing the major barriers they face in these processes.

Keywords: international migration, immigrant doctors from Latin America, professional integration, barriers, Catalan and Spanish context.

Recebido para publicación en 25/08/2013.

Aceptado para publicación en 07/11/2013.

Received for publication in August, 25 ${ }^{\text {th }}, 2012$.

Accepted for publication in November, 07 ${ }^{\text {th }}, 2013$. 